\title{
Concentrações de poluentes atmosféricos no Rio de Janeiro em relação a normas nacionais e internacionais
}

\author{
Eduardo Monteiro Martins ${ }^{\text {'; }}$ Aline Ribeiro Meireles ${ }^{\text {; }}$ Francisca Rodrigues Magalhaes 1; Josie \\ Batista Bastos Carvalho ${ }^{\text {; }}$ Maycon Maia Ribeiro ${ }^{l}$ \\ $\triangle$ edmmartins@gmail.com
}

1. Universidade do Estado do Rio de Janeiro, Rua São Francisco Xavier, 524, Rio de Janeiro, Brasil.

Histórico do Artigo:

Recebido: 26 de setembro de 2016

Aceito: 5 de abril de 2017

Publicado: 18 de julho de 2017

Resumo: Este trabalho avaliou as concentrações dos poluentes atmosféricos monitorados pelas estações automáticas de monitoramento da SMAC, comparando-nos aos padrões de qualidade do ar da legislação nacional e com as importantes legislações internacionais. 0s dados utilizados foram as médias horárias, as médias de três horas, de oito horas e de vinte e quatro horas, e compreendem o período entre 01/01/2012 e 31/12/2013. 0 ozônio $\left(0_{3}\right)$ foi o poluente que apresentou o maior número de violações em todas as estações quando a comparação foi

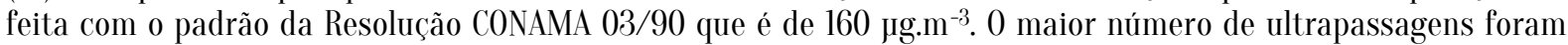
Bangu, Irajá e Campo Grande com, respectivamente, 215, 189 e 77 ultrapassagens. 0 cálculo do coeficiente de correlação e o resultado da análise de clusters mostrou que as estações de monitoramento da SMAC possuem dois grupos semelhantes.

Palavras-chave: Poluição do ar, C0NAMA 03/90, Ozônio, PMํ, Normas Internacionais.

\section{Air pollutants concentrations at Rio de Janeiro in relation to national and international laws}

\begin{abstract}
This work has made the assessment of air pollutants concentrations monitored by the SMAC automatic monitoring stations, comparing the concentration values obtained with the air quality standards of national legislation and of important international laws. The concentration data were provided during the period between 01/01/2012 to 12/31/2013. The frequency was supplied hourly averages, in addition to average three hours, eight hours and twenty-four hours. Ozone $\left(0_{3}\right)$ is the pollutant that showed the highest number of exceedances in all seasons when the comparison was made with the standard of CONAMA Resolution 03/90, which is $160 \mu \mathrm{g} . \mathrm{m}^{-3}$. The highest number of exceedances were in Bangu, Campo Grande and Irajá, respectively, 215, 189 and 77 exceedances. The calculation of the correlation coefficient and the result of the cluster analysis showed that the monitoring stations SMAC have two similar groups.

Keywords: Air pollution, CONAMA 03/90, Ozone, $\mathrm{PM}_{10}$, International Standards.
\end{abstract}




\title{
Concentraciones de polluentes aéreos en el Rio de Janeiro en relación con las leyes nacionales e internacionales
}

\begin{abstract}
Resumen: Este trabajo fue la evaluación de las concentraciones de contaminantes atmosféricos monitoreados por las estaciones automáticas de monitoreo de SMAC, comparando los valores de las concentraciones obtenidas con los estándares de calidad del aire y la legislación nacional de diversa legislación internacional importante. Se proporcionaron los datos de concentración durante el periodo comprendido entre el 01/01/2012 y el 31/12/2013. La frecuencia se suministra medias de una hora, los promedios de tres horas, ocho horas, y veinticuatro horas. El ozono $\left(0_{3}\right)$ es el contaminante que tuvo el mayor número de superaciones en todas las estaciones en que se hizo la comparación con el estándar de la Resolución CONAMA 03/90, que es de $160 \mu \mathrm{g} . \mathrm{m}^{-3}$. El mayor número de superaciones fueron Bangu, Irajá y Campo Grande, respectivamente, 215, 189 y 77 superaciones. El cálculo del coeficiente de correlación y el resultado de los análisis de agrupamiento mostró que las estaciones de vigilancia SMAC tienen dos grupos similares.

Palabras clave: Contaminación del aire, CONAMA 03/90, Ozono, PM10, Normas Internacionales.
\end{abstract}

\section{INTRODUÇÃO}

Nos últimos anos vêm aumentando as preocupações relacionadas às questões ambientais e em especial aos problemas relacionados à poluição atmosférica. Com o crescimento dos processos de urbanização e o consequente aumento das fontes de emissão de poluentes atmosféricos tende-se a elevar a degradação da qualidade do ar e os diferentes impactos negativos. Entre os problemas relacionados à saúde ocorre o agravamento das doenças cardiovasculares, respiratórias, neurológicas e diferentes tipos de cânceres. As crianças e idosos são 0 grupo da população mais suscetível à degradação da qualidade do ar (BRUNEKREEF; HOLGATE, 2002; GURJAR et. al., 2008; KAMPA; CASTANAS, 2008).

Os poluentes mais comumente estudados nas principais cidades do mundo são denominados poluentes regulamentados. Eles foram escolhidos pelos seus conhecidos danos à saúde da população e por estarem presentes em concentrações significativas. De uma maneira geral, estes poluentes são o monóxido de carbono ( $\mathrm{C} 0)$, dióxido de enxofre $\left(\mathrm{S}_{2}\right)$, dióxido de nitrogênio $\left(\mathrm{NO}_{2}\right)$, partículas inaláveis (PI ou PM10) e ozônio $\left(0_{3}\right)$ (GIANNOULI et al., 2011; SANTANA et al., 2011). 0 ozônio é um poluente que não é emitido diretamente na atmosfera por fontes móveis e estacionárias, portanto é considerado um poluente secundário formado por reações químicas na atmosfera. As reações de 1 a 3 mostram o processo de formação na troposfera sem a presença de compostos orgânicos voláteis (COVs) (ATKINSON, 2000). 


$$
\begin{gathered}
\mathrm{NO}_{2}+h w \rightarrow \mathrm{NO}+0\left({ }^{3} \mathrm{P}\right)(1) \\
O\left({ }^{3} \mathrm{P}\right)+\mathrm{O}_{2}+\mathrm{M} \rightarrow \mathrm{O}_{3}(2) \\
\mathrm{O}_{3}+\mathrm{NO} \rightarrow \mathrm{NO}_{2}+\mathrm{O}_{2}(3)
\end{gathered}
$$

Em atmosferas com presença dos COVs ocorre o desequilíbrio entre as etapas de formação e o consumo do ozônio acarretando um acúmulo deste poluente na troposfera. Esse desequilíbrio é ocasionado por duas etapas de oxidação dos hidrocarbonetos, onde ocorre a transformação de $\mathrm{N} 0$ para $\mathrm{NO}_{2}$, sem o consumo de ozônio, favorecendo os processos de formação em comparação com os processos de consumo.

Na presença dos COVs, o equilíbrio destas espécies é desestabilizado. Os COVs reagem com diferentes radicais que estão presentes na atmosfera como, por exemplo, o radical $0 \mathrm{H}$. A sequência das reações dos COVs é apresentada a seguir:

$$
\begin{gathered}
\mathrm{RH}+\mathrm{OH} \rightarrow \mathrm{R}+\mathrm{H}_{2} \mathrm{O}(4) \\
\mathrm{R}+\mathrm{O}_{2} \rightarrow \mathrm{RO}_{2}(5) \\
\mathrm{RO}_{2}+\mathrm{NO} \rightarrow \mathrm{RO}+\mathrm{NO}_{2}(6) \\
\mathrm{RO}+\mathrm{O}_{2} \rightarrow \mathrm{R}^{\prime} \mathrm{CHO}+\mathrm{HO}_{2}(7) \\
\mathrm{HO}_{2}+\mathrm{NO} \rightarrow \mathrm{OH}+\mathrm{NO}_{2}(8)
\end{gathered}
$$

Alguns destes radicais $\left(\mathrm{HO}_{2}\right.$ e $\mathrm{RO}_{2}$ ) reagem com o $\mathrm{NO}$ (reações 5 e 7), convertendo-o a $\mathrm{NO}_{2}$. 0 ozônio formado também é consumido pela reação 3 , mas devido ao aumento da conversão de $\mathrm{NO}$ a $\mathrm{NO}_{2}$, a formação de ozônio será elevada quando a taxa de fotólise do $\mathrm{NO}_{2}$ atingir um valor máximo (ATKINSON, 2000; SEINFELD et al., 1989).

Há muitos anos, o ozônio é um poluente que apresenta altas concentrações em diferentes cidades do mundo e conhecer os seus processos de formação é de fundamental importância para determinação de estratégias do controle de suas concentrações (ATKINSON, 2000; PARRISH et al., 2011). 
Concentrações de poluentes atmosféricos no Rio de Janeiro em relação a normas nacionais e internacionais

Além das elevadas concentrações de $0_{3}$, cidades com grandes frotas veiculares apresentam problemas com altas concentrações de material particulado de diferentes tamanhos aerodinâmicos, em especial as partículas inaláveis de $10 \mu \mathrm{m}\left(\mathrm{PM}_{10}\right)$ e partículas respiráveis de 2,5 ㅆm (PM2,5) (SINGH et al., 2014; VOORHES, 2014). Segundo a Organização Mundial de Saúde (0MS), o material particulado é responsável por diversos problemas na saúde, sendo um excelente indicador de correlação entre saúde pública e poluição atmosférica (0MS, 2005). Quanto menor o tamanho da partícula, mais agressiva ela é à saúde, pois irá penetrar mais profundamente no aparelho respiratório (HAUCK, 1998; SINGH et al., 2014).

Segundo a 0MS, cada país deve estabelecer seus próprios padrões de qualidade do ar de acordo com suas especificidades (0MS, 2005). A legislação brasileira que regulamenta os poluentes atmosféricos, bem como os seus valores máximos de concentrações é a resolução CONAMA 03/90 (BRASIL, 1990). Esta resolução não passou por atualização dos valores dos padrões de qualidade do ar desde a sua criação. As principais agências ambientais do mundo realizam atualizações dos seus valores limites com certa frequência. Podemos destacar algumas das últimas atualizações: Organização Mundial de Saúde (0MS) em 2005 (0MS, 2005); Environmental Protection Agency (EPA) em 2012 (EPA, 2012); a agência do estado da Califórnia (CARB) em 2007 (CARB, 2007); Normas Oficiais Mexicanas (NOM) em 2010 (MÉXICO, 2002; MÉXIC0, 2010), padrões de qualidade do ar da China em 2012 (CHINA, 2012); a Lei do Ar da Índia em 2009 (ÍNDIA, 2009); e da União Europeia, em 2008 (EUROPEAN COMMISSION, 2008). As mudanças foram consequências do desenvolvimento das cidades, do aumento de frota veicular, das concentrações observadas, melhoria dos equipamentos de controle das emissões, métodos de monitoramento e estudos dos impactos na saúde dos seres humanos. No ano de 2013, a Companhia de Tecnologia de Saneamento Ambiental (CETESB) passou a ter os seus próprios padrões de qualidade do ar (CETESB, 2013), assim como o Instituto Estadual do Ambiente (INEA), que regula a qualidade do ar do estado do Rio de Janeiro (INEA, 2013). A CETESB propôs seus padrões baseado nos valores recomendados pela OMS, porém com uma implementação gradativa, até que o valor final seja alcançado (CETESB, 2013).

A cidade do Rio de Janeiro é a região mais densamente povoada e que apresenta a segunda maior frota veicular do país e uma topografia bastante característica com influência da brisa do mar e montanhas que dificultam a dispersão dos poluentes. Desta forma, a poluição atmosférica é um problema que afeta a qualidade de vida da sua população e as altas concentrações dos poluentes devem ser monitoradas e controladas (SMAC, 2012). A rede de 
monitoramento de poluentes atmosféricos da cidade do Rio de Janeiro é composta pelas estações do Instituto Estadual do Ambiente (INEA) e pela Secretaria de Meio Ambiente da Cidade do Rio de Janeiro (SMAC) (SMAC, 2012; INEA, 2013). Os poluentes monitorados são os regulamentados pela resolução CONAMA 03/90 acrescidos dos hidrocarbonetos não metânicos (HCNM ou COVs) e do material particulado de tamanho aerodinâmico de 2,5 $\mu \mathrm{m}\left(\mathrm{PM}_{2,5}\right)$. A importância de se fazer o monitoramento dos COVs é que eles participam das reações de formação de $0_{3}$, portanto é fundamental o conhecimento das suas concentrações para os estudos cinéticos dos processos de formação do ozônio. 0 PM $_{2,5}$ já vem sendo monitorado e regulamentado nos Estados Unidos, na União Europeia e está presente na lista de poluentes atmosféricos que devem ser monitorados da OMS (OMS, 2005; CARB, 2007; EUROPEAN COMMISSION, 2008; EPA, 2012).

De acordo com a SMAC, a rede de monitoramento da Prefeitura do Rio de Janeiro opera desde 0 ano 2000, onde inicialmente eram monitorados na cidade do Rio de Janeiro somente as partículas inaláveis $\left(\mathrm{PM}_{10}\right)$, o dióxido de enxofre $\left(\mathrm{SO}_{2}\right)$ e o monóxido de carbono (C0) (SMAC, 2012). As estações estavam localizadas nos bairros do Centro, Copacabana, Tijuca e São Cristóvão. Além destas quatro estações fixas, o monitoramento era feito com uma estação móvel que ainda fazia as medidas das concentrações de $0_{3}$, $\mathrm{HCNM}$ e $\mathrm{NO}_{\mathrm{x}}$. A estação móvel realizava o monitoramento em curtos períodos de tempo e em diferentes locais da cidade. No final do ano de 2011, esta rede foi ampliada para mais quatro estações fixas. As novas estações fixas estão instaladas nos bairros de Bangu, Campo Grande, Guaratiba e Irajá. As novas estações realizam o monitoramento do $\mathrm{PM}_{10}, \mathrm{SO}_{2}, \mathrm{CO}, \mathrm{O}_{3}, \mathrm{HCNM}, \mathrm{NO}_{\mathrm{x}}$ e $\mathrm{PM}_{2,5}$.

Sousa et al. (2012) fez a avaliação dos dados monitorados pela SMAC entre os anos de 2000 e 2005. Neste trabalho foram avaliadas as concentrações de C0, $\mathrm{PM}_{10}$ e $\mathrm{SO}_{2}$ nas estações do Centro e São Cristóvão. Quando os dados foram comparados com a legislação europeia foi observado um grande número de ultrapassagens para o $\mathrm{PM}_{10}$ nos seis anos de dados analisados (SOUSA et al., 2012). Não foram encontrados outros trabalhos na literatura comparando as concentrações dos poluentes atmosféricos regulamentados nas principais cidades brasileiras com a legislação internacional.

Este trabalho tem como objetivo fazer a avaliação das concentrações dos poluentes atmosféricos monitorados pelas estações automáticas de monitoramento da SMAC, comparar os valores obtidos com os valores vigentes das legislações brasileiras e internacionais e verificar a semelhança entre as estações da SMAC. 
Concentrações de poluentes atmosféricos no Rio de Janeiro em relação a normas nacionais e internacionais

\section{METODOLOGIA}

0 monitoramento da qualidade do ar é definido através de uma seleção de poluentes atmosféricos que são observados com frequência e que sejam impactantes na saúde da população e ao equilíbrio do meio ambiente. 0 monitoramento da qualidade do ar na cidade do Rio de Janeiro é realizado, pela SMAC, nos bairros de Bangu, Campo Grande, Centro, Copacabana, Guaratiba, Irajá, São Cristóvão e Tijuca. A distribuição espacial das estações da rede de monitoramento da Prefeitura do Rio de Janeiro está apresentada na Figura 1.

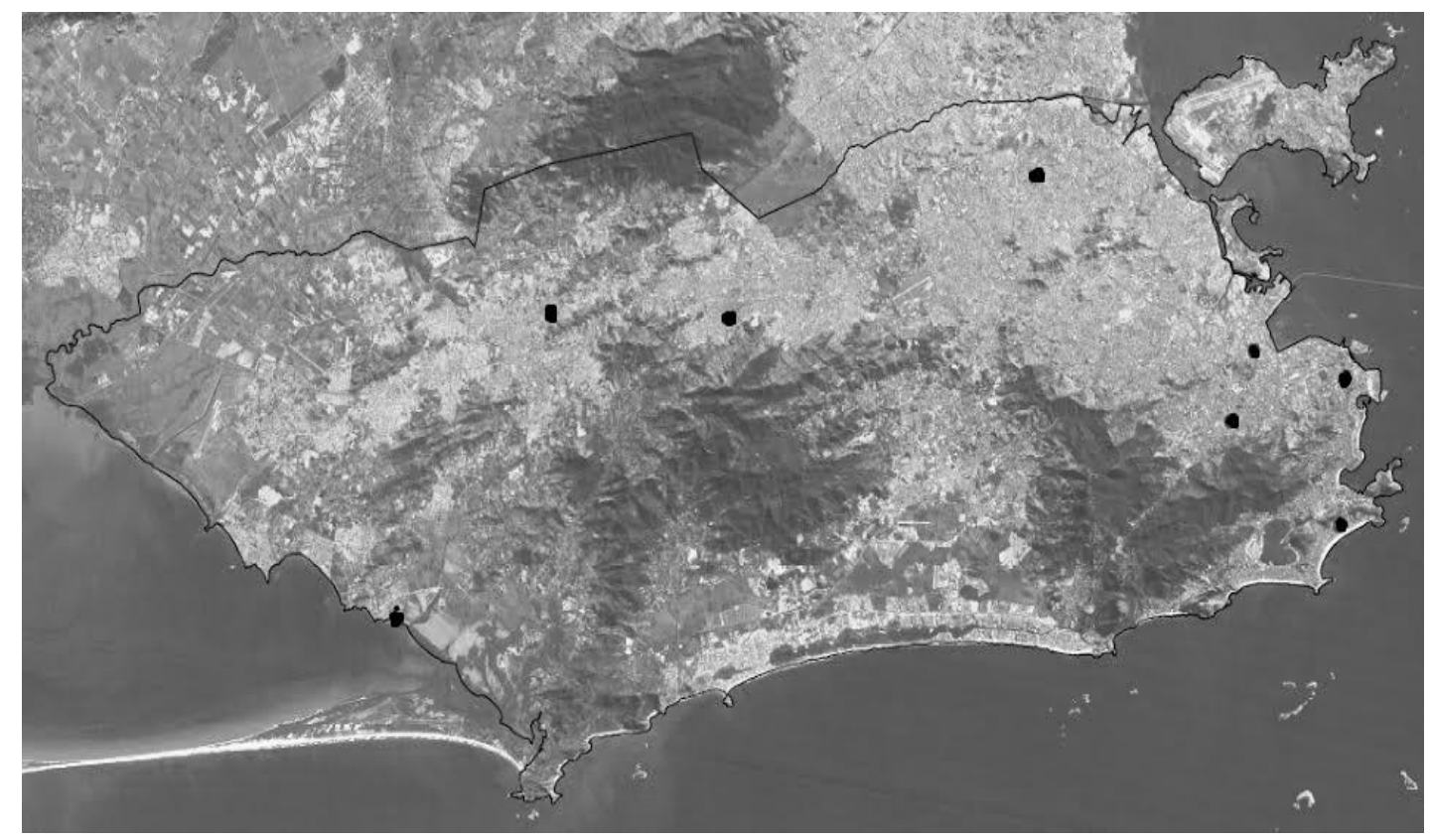

Figura 1. - Distribuição espacial da rede de monitoramento da prefeitura da cidade do Rio de Janeiro

0 monitoramento é feito de forma contínua e enviado para o banco de dados da Secretaria de Meio Ambiente da Cidade do Rio de Janeiro por telemetria. Os dados das concentrações dos poluentes foram fornecidos pela prefeitura em médias horárias, médias de três horas, oito horas e vinte quatro horas. 0 período dos dados fornecidos pela SMAC foi dos anos de 2012 e 2013. Na Tabela 1 é apresentada a configuração dos poluentes que são monitorados em cada uma das estações da SMAC. 
Tabela 1 - - Poluentes monitorados nas diferentes estações da rede de monitoramento da SMAC

\begin{tabular}{llllllll}
\hline Bairro/Poluente & $\mathrm{CO}$ & $\mathrm{SO}_{2}$ & $\mathrm{O}_{3}$ & $\mathrm{PM}_{10}$ & $\mathrm{NO}_{2}$ & $\mathrm{PM}_{2,5}$ & $\mathrm{HCNM}$ \\
\hline Bangu & $\mathrm{X}$ & $\mathrm{X}$ & $\mathrm{X}$ & $\mathrm{X}$ & $\mathrm{X}$ & 0 & $\mathrm{X}$ \\
Copacabana & $\mathrm{X}$ & $\mathrm{X}$ & $\mathrm{X}$ & $\mathrm{X}$ & 0 & 0 & 0 \\
Centro & $\mathrm{X}$ & $\mathrm{X}$ & $\mathrm{X}$ & $\mathrm{X}$ & 0 & 0 & 0 \\
Campo Grande & $\mathrm{X}$ & $\mathrm{X}$ & $\mathrm{X}$ & $\mathrm{X}$ & $\mathrm{X}$ & 0 & $\mathrm{X}$ \\
Irajá & $\mathrm{X}$ & $\mathrm{X}$ & $\mathrm{X}$ & $\mathrm{X}$ & $\mathrm{X}$ & $\mathrm{X}$ & $\mathrm{X}$ \\
Tijuca & $\mathrm{X}$ & $\mathrm{X}$ & $\mathrm{X}$ & $\mathrm{X}$ & 0 & 0 & 0 \\
São Cristóvão & $\mathrm{X}$ & $\mathrm{X}$ & $\mathrm{X}$ & $\mathrm{X}$ & 0 & 0 & 0 \\
Guaratiba & 0 & 0 & $\mathrm{X}$ & $\mathrm{X}$ & 0 & 0 & 0 \\
\hline
\end{tabular}

X: Poluente monitorado, 0: Poluente não monitorado, C0: Monóxido de Carbono, S02: Dióxido de Enxofre, 03: Ozônio, PM10: Partículas de até 10 micrometros, PM2,5: Partículas de até 2,5 micrmetros, HCNM: Hidrocarbonetos não metânicos.

A partir dos dados de concentração dos poluentes foram feitos estudos para determinação do perfil de comportamento médio horário para cada poluente em cada estação. Os dados de concentração foram comparados com a legislação nacional e internacional. Como padrões internacionais foram escolhidos o padrão nacional dos Estados Unidos (EPA), o padrão estadual da agência da Califórnia (CARB), Comunidade Européia (EU), Organização Mundial da Saúde (OMS), México (NOM), Índia e China para verificar a frequência de ultrapassagens das concentrações medidas. Na Tabela 2 são mostrados os padrões de concentração dos poluentes atmosféricos para a legislação nacional e para as legislações internacionais selecionadas para este trabalho. 
Concentrações de poluentes atmosféricos no Rio de Janeiro em relação a normas nacionais e internacionais

Tabela 2 - Poluentes legislados no CONAMA 03/90 e normas internacionais.

\begin{tabular}{|c|c|c|c|c|c|c|c|c|c|}
\hline $\begin{array}{c}\text { Legislação } \\
\text { / Poluentes } \\
\left(\mu \mathrm{gg} / \mathrm{m}^{3}\right)\end{array}$ & $\begin{array}{c}\text { CONAMA } \\
\text { 03/90 }\end{array}$ & $\begin{array}{c}\text { CETESB } \\
59.113 / 2013\end{array}$ & EPA & CARB & CHINA & ÍNDIA & OMS & NOM & $\mathbf{E U}$ \\
\hline $\begin{array}{l}\text { Partículas } \\
\text { inaláveis - } \\
\text { PM }_{10} \\
(24 h s)\end{array}$ & 150 & 120 & 150 & 50 & 150 & 100 & 50 & 120 & 50 \\
\hline $\begin{array}{l}\text { Partículas } \\
\text { inaláveis - } \\
\text { PM }_{10} \\
\text { (MAA) }\end{array}$ & 50 & 40 & - & 20 & 70 & 60 & 20 & 50 & 40 \\
\hline $\begin{array}{l}\text { PTS } \\
\text { (24hrs) }\end{array}$ & 80 & 80 & - & - & - & - & - & - & - \\
\hline $\begin{array}{l}\mathrm{SO}_{2} \\
(1 \mathrm{~h})\end{array}$ & - & & 196 & - & - & - & - & - & 350 \\
\hline $\begin{array}{l}\mathrm{SO}_{2} \\
(24 \mathrm{hrs})\end{array}$ & 365 & 60 & - & 105 & 150 & 80 & 20 & 288 & 125 \\
\hline $\begin{array}{l}\mathrm{SO}_{2} \\
\text { (MAA) }\end{array}$ & 80 & 40 & - & - & 60 & 50 & - & 66 & - \\
\hline $\begin{array}{l}\text { CO } \\
\text { (1h) }\end{array}$ & 40.000 & 40.000 & 40000 & 23000 & 10000 & 40000 & - & - & - \\
\hline $\begin{array}{l}\text { CO } \\
(8 \mathrm{hs})\end{array}$ & 10.000 & 10.000 & 10000 & 10000 & 10000 & 10000 & 10000 & 12595 & 10000 \\
\hline $\begin{array}{l}\mathbf{O}_{3} \\
(1 \mathrm{~h})\end{array}$ & 1601 & & - & 180 & 200 & 180 & - & 220 & - \\
\hline $\begin{array}{l}\mathrm{O}_{3} \\
(\mathbf{8 h})\end{array}$ & - & 140 & - & - & 160 & 100 & 100 & 160 & 120 \\
\hline $\begin{array}{l}\mathrm{NO}_{2} \\
(\mathbf{l h})\end{array}$ & 320 & 260 & 188 & 339 & 200 & - & 200 & 395 & 200 \\
\hline $\begin{array}{l}\mathrm{NO}_{2} \\
\text { (MAA) }\end{array}$ & 100 & 60 & 100 & 57 & 40 & 40 & 40 & - & 40 \\
\hline
\end{tabular}




\section{RESULTADOS E DISCUSSÃ0}

A avaliação do perfil de comportamento para os poluentes e suas características é apresentada na Figura 2. É possível observar que não existe uma estação onde as concentrações de monóxido de carbono sejam predominantemente mais altas. 0 ozônio, poluente secundário e de formação fotoquímica, apresenta as maiores concentrações entre às 13:00 h e às 16:00 h. Avaliando o perfil de concentração do 03, as duas estações com as mais altas concentrações médias são Bangu e Irajá. Sem a incidência de luz solar à noite, as reações de consumo de ozônio prevalecem em relação às reações de formação de ozônio que ocorrem durante o dia. Desta forma era esperado que as concentrações diminuíssem neste período.

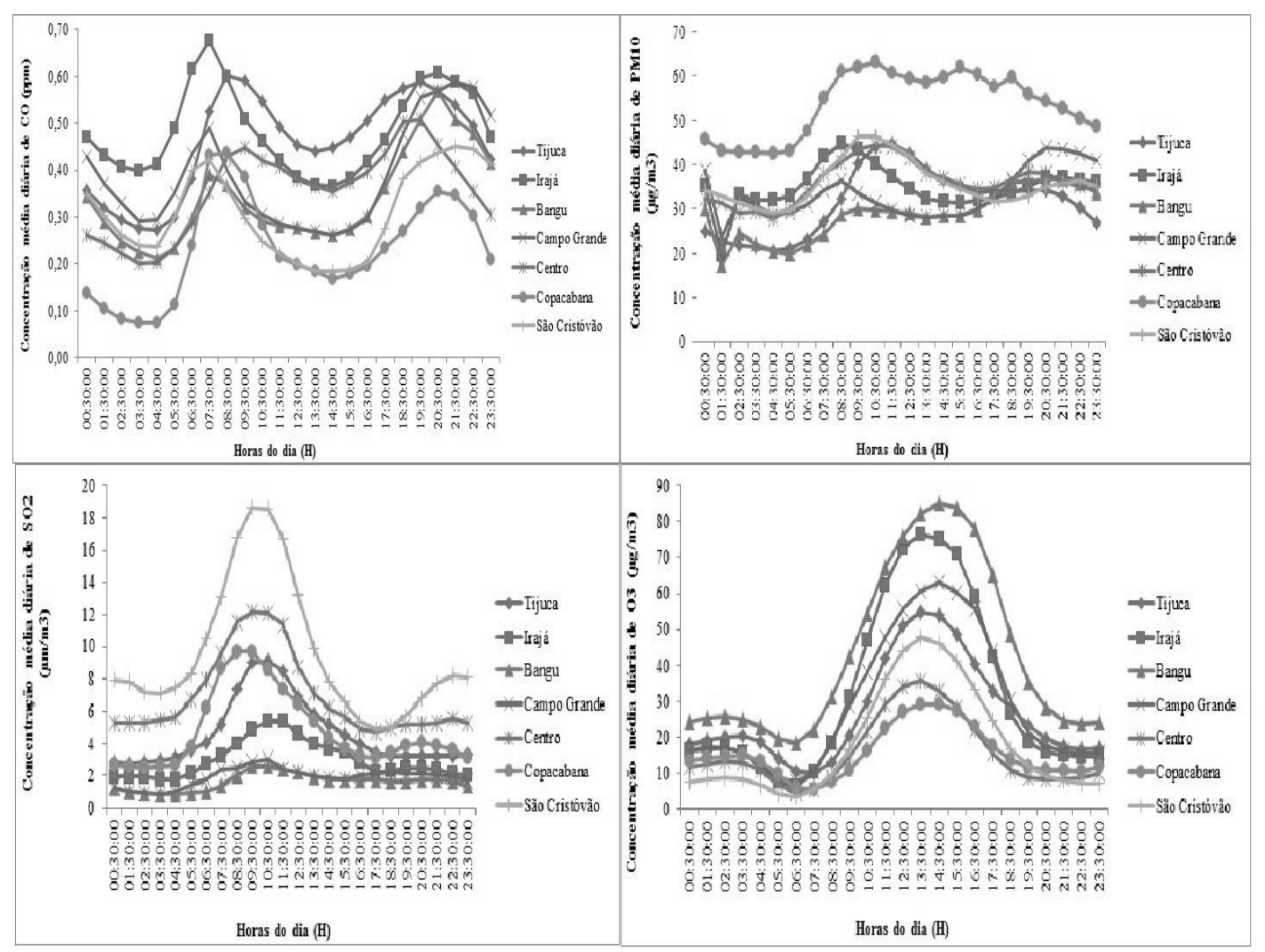

Figura 2. - Concentrações médias diária dos poluentes C0, PM10, S02 e 03 para a rede de monitoramento da SMAC nos anos de 2012 e 2013.

Quando a avaliação é feita para os demais poluentes, podemos observar que a estação de Copacabana apresenta o perfil médio mais elevado para as partículas inaláveis do que as demais estações. Já para o dióxido de enxofre, a estação que apresenta o perfil médio mais alto é a estação de São Cristóvão. Esta estação encontra-se próxima da Avenida Brasil, da Linha 
Concentrações de poluentes atmosféricos no Rio de Janeiro em relação a normas nacionais e internacionais

Vermelha, da Rodoviária Novo Rio e o do Porto da Cidade do Rio de Janeiro. Essa proximidade de locais com altas nas emissões de veículos a diesel justifica as concentrações mais altas nessa estação.

Comparando as concentrações medidas com as diferentes legislações, o ozônio $\left(0_{3}\right)$ foi 0 único poluente que apresentou ultrapassagens em todas as estações, exceto Tijuca, quando a comparação foi feita com o padrão da Resolução CONAMA 03/90 que é de $160 \mu \mathrm{g} \mathrm{m} \mathrm{m}^{-3}$. Sendo também o poluente que apresentou o maior número de violações nas demais legislações avaliadas. Comparando com a Resolução CONAMA, os bairros que apresentaram o maior número de ultrapassagens foram Bangu, Irajá e Campo Grande com, respectivamente, 215, 189 e 77 ultrapassagens. Já os bairros de São Cristóvão, Centro e Copacabana apresentaram 19, 4 e 1 violações respectivamente. A Resolução CONAMA permite que o padrão de qualidade do ar seja violado uma única vez ao ano. A maior concentração de ozônio foi observada na estação de Campo Grande, com o valor de 307,76 $\mu \mathrm{g} . \mathrm{m}^{-3}$, sendo 191,85\% acima do padrão de qualidade do ar. 0 número de violações das concentrações de ozônio para as diferentes legislações internacionais e para a Resolução CONAMA para os padrões de uma hora está apresentado na Figura 3.

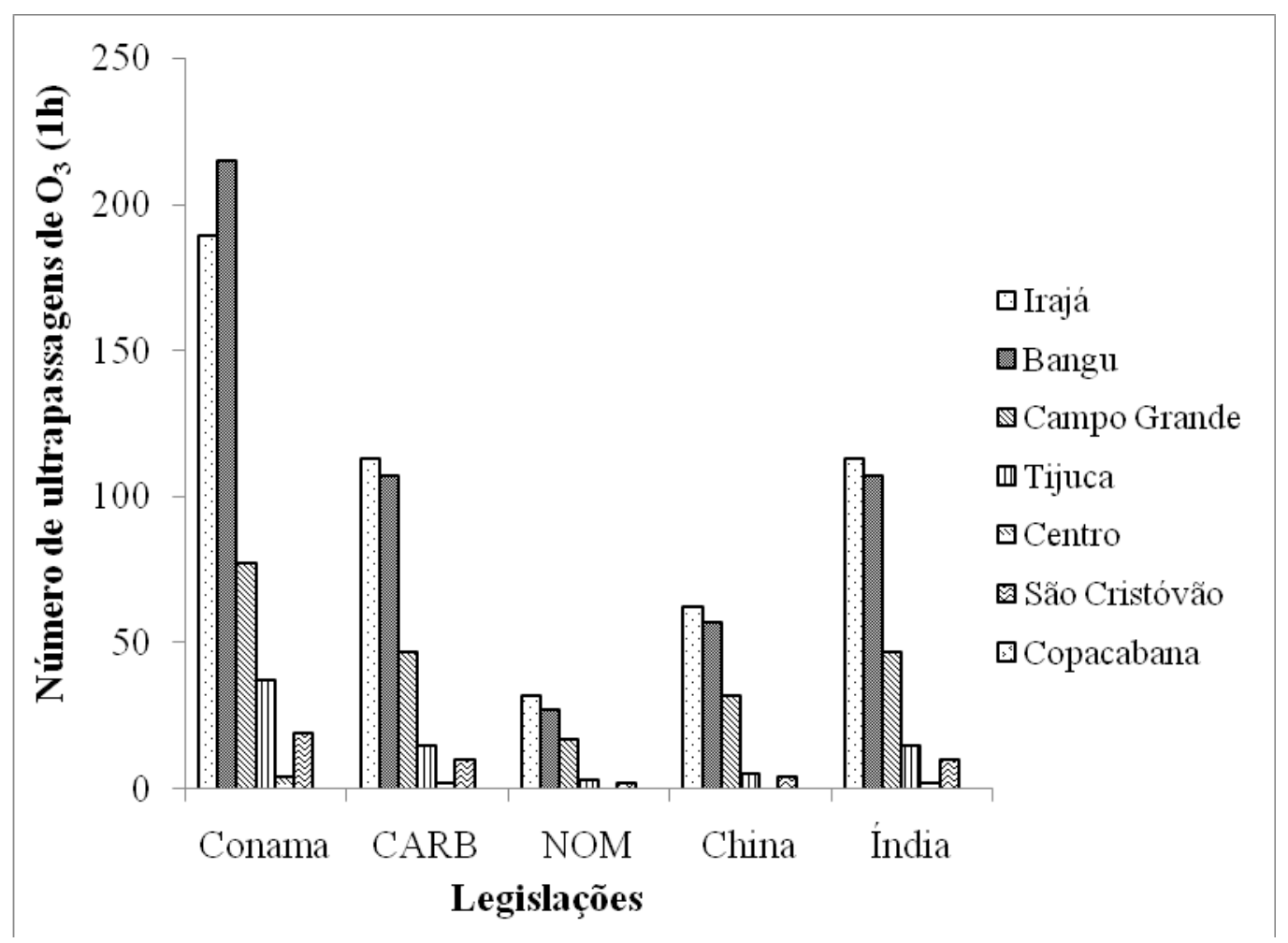

Figura 3 - Ultrapassagens das concentrações de 03 aos padrões internacionais e nacional durante os anos de 2012-2013. 
Comparando os padrões de qualidade do ar adotados na legislação nacional com os das legislações internacionais selecionadas, o valor do CONAMA para a concentração do ozônio para o padrão de uma hora é o mais restritivo. A legislação mexicana é a mais permissiva com um valor máximo de concentração de $220 \mu \mathrm{g}$. $\mathrm{m}^{-3}$. Mesmo quando as concentrações medidas são comparadas com os valores dos padrões mexicanos, o ozônio viola o padrão adotado. As três estações que mais apresentaram violações para a legislação Mexicana foram Irajá, Bangu, e Campo Grande com 32, 27 e 17 violações respectivamente.

As legislações internacionais da EPA, CARB, EU, Índia, China e OMS possuem valor padrão para concentração máxima média em oito horas. 0 padrão mais restritivo para o padrão de oito horas é da OMS e da Índia, ambos de $100 \mu \mathrm{g} . \mathrm{m}^{-3}$. Quando os valores monitorados são comparados com estes padrões ocorrem violações para todas as estações exceto para a estação de Copacabana e Tijuca. 0 ponto de monitoramento com maior número de ultrapassagens foi 0 bairro de Bangu, com 112 ultrapassagens no período dos anos de 2012 e 2013. A estação de Irajá apresentou 77 violações a este padrão para o mesmo período. A concentração adotada pela CETESB para o padrão de 8 horas de ozônio é de $140 \mu \mathrm{g} . \mathrm{m}^{-3}$. Ao fazer a comparação com estes padrões é possível observar violações nas estações de Irajá, Bangu e Campo Grande com respectivamente 17,12 e 7 violações.

Outro poluente que atinge e ultrapassa os valores de concentração dos padrões de qualidade do ar aplicados nesse estudo é o material particulado menor que $10 \mu \mathrm{m}$ ( $\left.\mathrm{PM}_{10}\right)$. Quando a comparação é feita com os padrões nacionais é observado apenas uma violação na estação de Irajá e outra violação na estação do Centro. Ao comparar com os padrões propostos pelas agências internacionais, todas as estações apresentam violação aos padrões. A estação de Copacabana apresenta 406 ultrapassagens ao padrão de qualidade do ar proposto pela CARB, OMS e UE, que é de $50 \mu g . \mathrm{m}^{-3}$. A segunda estação com maior número de ultrapassagens é a estação de São Cristóvão, com 146 ultrapassagens. A localização destas estações contribui para o resultado, uma vez que a estação de Copacabana está na direção de um ponto de ônibus e a estação de São Cristóvão é próxima ao terminal rodoviário, Avenida Brasil, da Linha Vermelha e do porto da Cidade do Rio de Janeiro, o que aumenta as emissões deste poluente devido ao maior número de veículos a diesel. Para as legislações da China, Índia e México também ocorrem violações para o $\mathrm{PM}_{10}$, entretanto elas são bem menores uma vez que os padrões de qualidade do ar adotados são mais permissivos. A CETESB sugere um valor de $120 \mu \mathrm{g} . \mathrm{m}^{-3}$, ao comparar as 
Concentrações de poluentes atmosféricos no Rio de Janeiro em relação a normas nacionais e internacionais

concentrações medidas com este valor foi observada apenas uma violação, que foi medida na estação do Centro.

As partículas respiráveis ou $\mathrm{PM}_{2,5}$ não são regulamentas no Brasil, entretanto são monitoradas pela estação localizada no Bairro de Irajá. Entre as legislações internacionais vigentes, os valores mais restritivos para o $\mathrm{PM}_{2,5}$ são para a $\mathrm{OMS}$ e EU, onde os valores limites são respectivamente de 20 e $25 \mu \mathrm{g} . \mathrm{m}^{-3}$. Para o padrão da 0MS, que é o mais restritivo ocorreram 123 ultrapassagens no período avaliado.

0 dióxido de enxofre apresentou violação apenas quando a comparação foi feita ao limite estabelecido pela Organização Mundial de Saúde que é de $20 \mu \mathrm{g} . \mathrm{m}^{-3}$ e a estação de São Cristóvão foi a que apresentou o maior número de ultrapassagens, um total de 70 ultrapassagens no período de estudo. Atualmente, as concentrações médias de 24 horas são bem menores que 0 atual padrão de qualidade do ar propostos, que é de $365 \mu \mathrm{g} . \mathrm{m}^{-3}$ para 0 mesmo período. A maior média de 24 horas medida neste período foi na estação de Campo Grande e o valor medido foi de $134,87 \mu g . \mathrm{m}^{-3}$, que é apenas $37 \%$ do atual padrão de qualidade do ar.

0 dióxido de nitrogênio não apresentou violações para o padrão CONAMA e apresentou quatro violações na estação de Irajá para o padrão proposto pela EPA. 0 monóxido de carbono não apresentou nenhuma violação para nenhum padrão e para nenhuma legislação estudada. Sendo que as concentrações máximas de monóxido de carbono medidas nas estações ficaram muito abaixo do padrão de todas as legislações.

Na Tabela 3 é apresentada a matriz de correlação das concentrações de ozônio nas diferentes estações de monitoramento da SMAC. É possível observar correlações maiores que 0,7, que indicam correlações fortes e positivas e mostrando como as estações de monitoramento se relacionam em função das concentrações de ozônio. 0 mais alto coeficiente de correlação foi observado para as estações da Tijuca e São Cristóvão que estão distantes uma da outra em aproximadamente $5 \mathrm{~km}$, apresentando semelhantes processos de emissão e de dispersão dos poluentes. A correlação entre as estações do Centro e de Guaratiba foi a mais baixa $(0,54)$, sendo que estas estações estão distantes aproximadamente $60 \mathrm{~km}$. 
Tabela 3 - - Coeficiente de correlação entre os dados 03 de cada estação

\begin{tabular}{lcccccccc}
\hline \multicolumn{7}{c}{ COEFICIENTE DE CORRELAÇÃO DAS CONCENTRAÇÕES DE OZÔNIO } \\
Variáveis & Centro & Copacabana & $\begin{array}{c}\text { São } \\
\text { Cristóvão }\end{array}$ & Tijuca & Irajáa & Bangu & $\begin{array}{c}\text { Campo } \\
\text { Grande }\end{array}$ & $\begin{array}{c}\text { Pedra de } \\
\text { Guaratiba }\end{array}$ \\
\hline Centro & 1 & & & & & & & \\
Copacabana & 0,76 & 1 & & & & & & \\
São Cristóvão & 0,81 & 0,7 & 1 & & & & & \\
Tijuca & 0,77 & 0,7 & 0,86 & 1 & & & & \\
Irajá & 0,73 & 0,66 & 0,83 & 0,79 & 1 & & & \\
Bangu & 0,61 & 0,63 & 0,71 & 0,68 & 0,8 & 1 & & \\
Campo Grande & 0,61 & 0,61 & 0,75 & 0,7 & 0,82 & 0,83 & 1 & 1 \\
\hline $\begin{array}{l}\text { Pedra de } \\
\text { Guaratiba }\end{array}$ & 0,54 & 0,56 & 0,67 & 0,62 & 0,7 & 0,79 & 0,76 & 1 \\
\hline
\end{tabular}

0 cálculo do coeficiente de correlação de Pearson foi feito para as partículas inaláveis e para o monóxido de carbono. As correlações obtidas para o monóxido de carbono foram similares às obtidas para o ozônio, entretanto para as partículas inaláveis foram obtidos baixos coeficientes de correlação. A correlação obtida entre a estação da Tijuca e de São Cristóvão foi de 0,44, sendo este o par de estações que obteve a correlação mais alta para o ozônio e para 0 monóxido de carbono.

A análise de cluster apresentou como resultado a formação de dois grupos prioritários. Um grupo formado pelas estações de Pedra de Guaratiba, Bangu, Irajá e Campo Grande, o outro grupo é formado pelas estações da Tijuca, Centro, Copacabana e São Cristóvão. Esses dois grupos representam as quatro estações que foram instaladas em 2011 e o outro grupo é formado pelas primeiras quatro estações da rede de monitoramento da SMAC. 0 conjunto das estações do Centro, Tijuca, São Cristóvão e Copacabana são próximas entre si e apresentam comportamento similar devido as fontes de emissão. Na área de influência dessas estações circulam menos veículos a diesel e a idade da frota é mais nova quando comparada com o grupo das estações que estão localizadas ao longo da Avenida Brasil. As estações localizadas ao longo da Avenida Brasil (Irajá, Campo Grande, Bangu e Guaratiba) sofrem uma maior influência de emissões de veículos a diesel e uma frota veicular mais antiga quando comparada com as quatro estações do outro grupo (Pires, 2005). Os grupos formados mostram uma menor distância de ligação para o par de estação Tijuca/São Cristóvão confirmando a forte ligação para estas estações quando o ozônio é o composto que está sendo avaliado. 0 dendrograma obtido na análise de cluster para a concentração de ozônio nas diferentes estações está mostrado na Figura 4 
Concentrações de poluentes atmosféricos no Rio de Janeiro em relação a normas nacionais e internacionais

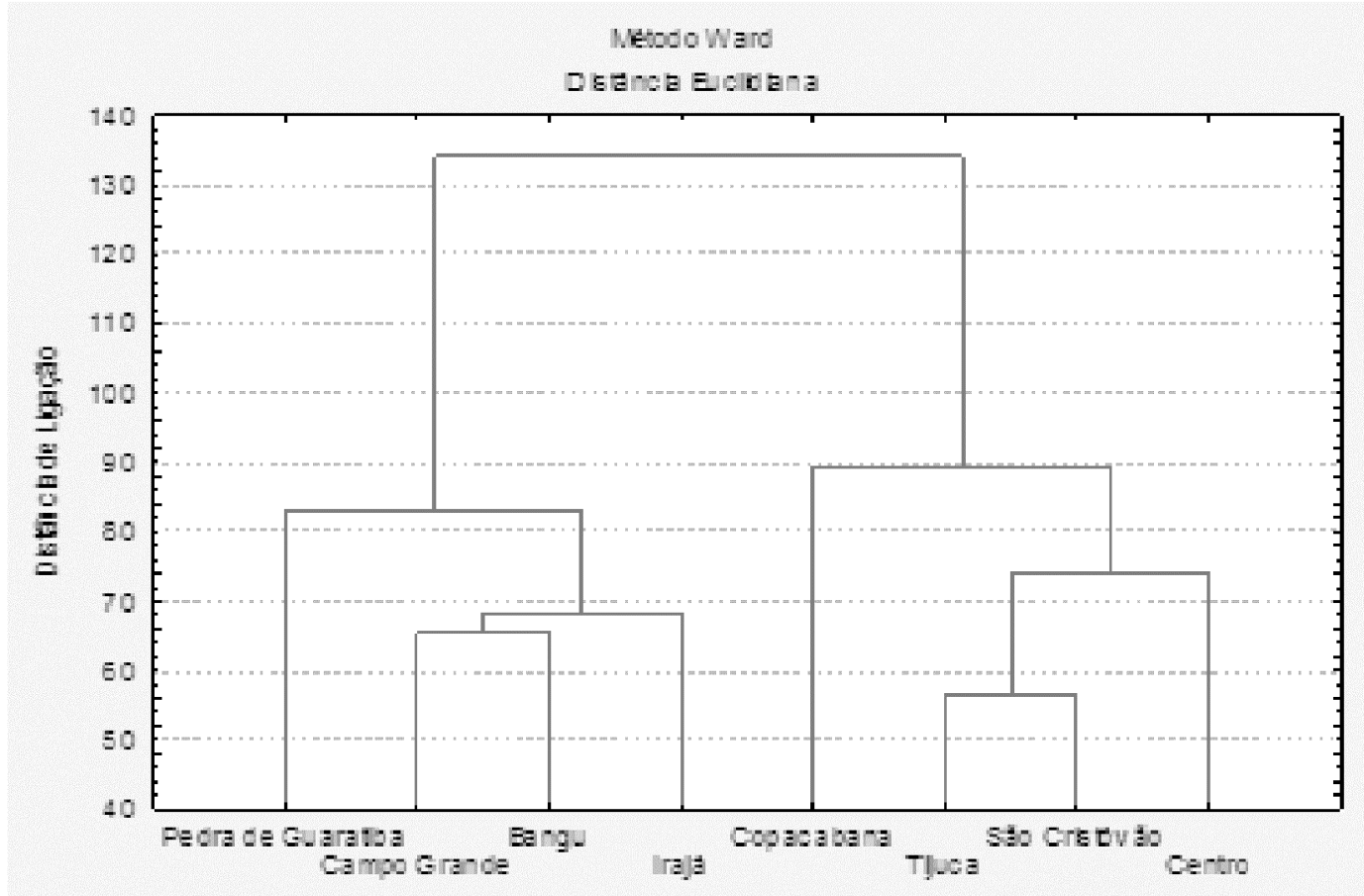

Figura 4 - Análise de Cluster entre os dados de concentração 03 para cada estação da rede de monitoramento da SMAC.

Em função dos resultados de monitoramento de dois anos nas estações da cidade do Rio de Janeiro e dos valores máximos observados foi proposta, na tabela 4, uma atualização aos valores limites da resolução CONAMA 03/90. É importante ressaltar que esta proposta está levando em consideração as concentrações monitoradas na cidade do Rio de Janeiro. Além da alteração dos valores do padrão dos poluentes que já são contemplados pela CONAMA 03/90 é sugerido um segundo padrão para o ozônio, sendo proposto um padrão de $100 \mu \mathrm{g} . \mathrm{m}^{3}$ em médias de 8 horas. Isto se deve ao fato do ozônio apresentar o maior número de violações em todas as estações e ser um poluente que merece um acompanhamento especial das suas concentrações. É proposto ainda um padrão de 24 horas e a média aritmética anual (MAA) para o $\mathrm{PM}_{2,5}$, além da inclusão do composto benzo(a)pireno. 
Tabela 4 - Proposta de alterações nos padrões da qualidade do ar da Resolução CONAMA 03/90

\begin{tabular}{|c|c|}
\hline Poluentes & Valor proposto $\left(\mathrm{\mu g} / \mathrm{m}^{3}\right)$ \\
\hline Partículas inaláveis - $\mathrm{PM}_{10}$ (24hs) & 50 \\
\hline Partículas inaláveis - $\mathrm{PM}_{10}$ (MAA) & 20 \\
\hline $\mathrm{SO}_{2}$ (24hs) & 60 \\
\hline CO (lh) & 40.000 \\
\hline C0 (8hs) & 10.000 \\
\hline $\mathrm{O}_{3}(\mathrm{lh})$ & 160 \\
\hline $\mathrm{NO}_{2}(\mathrm{lh})$ & 200 \\
\hline $\mathrm{NO}_{2}$ (MAA) & 40 \\
\hline \multicolumn{2}{|l|}{ Novos padrões } \\
\hline $0_{3}(8 \mathrm{~h})$ & 100 \\
\hline Material particulado fino $\mathrm{PM}_{2,5}$ (24hs) & 25 \\
\hline Material particulado fino $\mathrm{PM}_{2,5}(\mathrm{MAA})$ & 10 \\
\hline Benzo(a)pireno & 0,001 \\
\hline
\end{tabular}

\section{CONCLUSÃ0}

A discussão de novos padrões de qualidade do ar é fundamental uma vez que a legislação nacional não apresenta modificações desde sua criação. Poucos trabalhos comparam as concentrações de poluentes atmosféricos de cidades brasileiras com os padrões internacionais de qualidade do ar. Essas comparações permitem que uma nova interpretação da qualidade do ar das cidades brasileiras seja feita.

Na cidade do Rio de Janeiro, os dois poluentes que merecem maior atenção são o ozônio e o $\mathrm{PM}_{10} 0$ ozônio foi o poluente que apresentou o maior número de ultrapassagens em todas as legislações e o $\mathrm{PM}_{10}$ apresentou diversas ultrapassagens quando comparado com os padrões da CARB, OMS e UE. Para uma boa gestão da qualidade do ar é fundamental realizar o monitoramento dos poluentes atmosféricos, uma vez que podem servir como base para atualização da legislação brasileira.

A partir dos resultados obtidos, foi proposta a atualização da legislação nacional CONAMA03/90. Entre as mudanças/inclusões estão o padrão para o ozônio em média de 8 horas, onde propôs-se a concentração de $100 \mu \mathrm{g} . \mathrm{m}^{-3}$, de acordo com o estabelecido pela OMS. 0 PM 2,5 é 
Concentrações de poluentes atmosféricos no Rio de Janeiro em relação a normas nacionais e internacionais

um poluente que não está presente na CONAMA 03/90, o qual propôs-se o padrão de 25 Hg.m ${ }^{-3}$ para 24 horas e $10 \mu g . \mathrm{m}^{-3}$ como média aritmética anual, de acordo com o padrão da OMS.

A análise estatística multivariada indicou a existência de dois grupos de estações com comportamentos similares. 0 primeiro grupo é formado pelas estações da rede de monitoramento que estava em operação desde o ano 2000. 0 outro grupo é formado pelas estações que entraram em operação no ano de 2011.

\section{REFERÊNCIAS}

ATKINSON, R. Atmospheric chemistry of VOCs and N0x. Atmospheric Environment,2000, 34, 2063.

BRASIL. Resolução CONAMA nº 03 de 28 de junho de 1990. Dispõe sobre padrões de qualidade do ar, previstos no PRONAR. Brasília - DF, 1990.

BRUNEKREEF, B.; HOLGATE, S. T. Air pollution and health. The Lancet, 2002, 360, 1233.

CARB. California Govern. ARB missions and Goals. 2007.

CETESB. Decreto $\mathrm{n}^{0} 59.113$ de 23 de abril de2013. Estabelece novos padrões de qualidade do ar e dá providências correlatas. São Paulo - SP, 2013.

CHINA. Air Quality Standards. 2012.

EPA. National Ambient Air Quality Standards (NAAQS). Estados Unidos da América, 2012.

EUROPEAN COMMISSION. Air Quality Standards. 2012.

GIANNOUlI, M.; MOUSSIOPOUlOS, N.; KALOGNOMOU, E. A.; SAMARASS.; FIALA, J.; MELLIOS, G. Impact of European emission control strategies on urban and local air quality. Atmospheric Environment, 2011, 45, 4753.

GURJAR, B.R.; BUTLER, T.M.; LAWRENCE, M.G.; LELIEVELD, J. Evaluation of emissions and air quality in megacities. Atmospheric Environment, 2008, 42, 1593.

HAIR, J. F., ANDERSON, R. E., TALHAM, R. L., BLACK, W. C. Análise multivariada de dados, 5a ed., Bookman: Porto Alegre, 2005 .

HAUCK, H. Revision of ambiente air quality standards for PM? Toxicology Letters, 1998, 96, 269.

INDIA. Air Quality Standards. 2009.

INEA. Relatório da qualidade do ar do estado do Rio de Janeiro - Ano base 2010-2011. 2013.

KAMPA, M.; CASTANAS, E. Human health effects of air pollution. Environmental Pollution,2008, 15, 362.

MÉXICO. Modificacion a la norma oficial mexicana N0M-020-SSAl-1993, Salud ambiental. Criterios para evaluar la calidad del aire ambiente con respecto al ozono (03). 2002.

MÉXICO. Air Quality Standards. 2010. 
OMS. Guias de cualidad del aire de la 0MS relativas al material particulado, el ozone, el dióxido de nitrogênio, y el dióxido de azufre. Actualización mundial2005. 2005.

PARRISH, D. D; SINGHB, H. B; MOLINAC, L.; MADRONICHD, S. Air quality progress in North American megacities: A review. Atmospheric Environment, 2011, 45, 7015.

PIRES, D. 0. Inventário de emissões atmosféricas de fontes estacionárias e sua contribuição para a poluição do ar na região metropolitana do Rio de Janeiro. Rio de Janeiro: Universidade Federal do Rio de Janeiro, 2005.

RI0 DE JANEIR0. SMAC. Relatório da Rede MonitorAr Rio 2011-2012. Qualidade do Ar na Cidade do Rio de Janeiro. Prefeitura do Rio de Janeiro, 2012.

SANTANA, E.; CUNHA, K. B.; FERREIRA, A. L.; ZAMBONI, A. Padrões de qualidade do ar: experiência comparada Brasil, EUA e União Européia. Instituto de Energia e Meio Ambiente: São Paulo, 2012.

SEINFELD, J.H.; SANTANA, E.; CUNHA, K.B; FERREIRA, A. L. Urbana air pollution: State of the Science. Science, 1989 , 243,745 .

SINGH, A.K.; SINGH, S.P.; KUMAR, G; DEO, B. Assessment on particulate pollution in Sindri after closure of Sindri unit of fertilizer Corporation of India/ (FCI). International Journal of Current Engineering and Technology, 2014, 4, 376.

SOUSA, S.I.V.; PIRES, J.C.M.; MARTINS, E.M.; FORTES, J.D.N.; ALVIM-FERRAZ, M.C.M.; MARTINS, F.G. Short-term effects of air pollution on respiratory morbidity at Rio de Janeiro - PART I: Air Pollution Assessment. Environment International, 2012, 44, 18.

VOORHES, A.S.;WANG, J.; WANG, C.; ZHA0, B.; WANG, S.; KAN, H. Public health benefits of reducing air pollution in Shanghai: a proof-of-concept methodology with application to BenMap. Science of the Total Environment, 2014, 485, 396. 